\title{
SUPERHARMONIC FUNCTIONS ON FOLIATIONS
}

\author{
S. R. ADAMS
}

\begin{abstract}
We use techniques from geometric analysis to prove that any positive, leafwise superharmonic, measurable function on a Riemannian measurable foliation with transverse invariant measure, finite total volume and complete leaves is, in fact, constant on a.e. leaf.
\end{abstract}

\section{INTRODUCTION}

In [G], L. Garnett considers foliations of compact manifolds in which each leaf is given a Riemannian structure. She proves:

Theorem [G, Theorem 1(b), p. 286]. Any bounded Borel function $h$ which is harmonic on each leaf must be constant on almost all leaves, relative to any finite harmonic measure.

We will only consider here the case of a transverse invariant measure and finite total volume where the "finite harmonic measure" of this theorem reduces to the transversal measure.

The theorem is remarkable because it could be the case that every leaf is isometric to hyperbolic space. Thus, by the axiom of choice, one could simply choose a bounded harmonic function on each leaf. The ergodic nature of the theorem is that these choices cannot be made in a Borel way.

Her proof uses probability theory and is quite elegant. Here we take a geometric analysis approach and prove

Theorem 5.1. Let $(M, \mathscr{F}, \mathscr{R})$ be a finite volume oriented measurable Riemannian foliation such that a.e. leaf is complete. Let $h: M \rightarrow(0, \infty)$ be a measurable function such that, for a.e. leaf $L \subseteq M$, the function $h \mid L$ is superharmonic on the Riemannian manifold $L$. Then $h \mid L$ is constant, for a.e. leaf $L \subseteq M$.

It is possible to remove the orientability assumption in the theorem by introducing the orientable double cover. A positive superharmonic function will lift to a positive superharmonic function in the double cover, where the Theorem 5.1 applies. Therefore the lift is constant on a.e. leaf and it follows that the same holds for the original function.

The proof of Theorem 5.1 is best understood as an evolution of theorems.

Received by the editors October 20, 1989 and, in revised form, January 5, 1990.

1980 Mathematics Subject Classification (1985 Revision). Primary 28D99, 53C21. 
First is the very simple

Proposition 0.1. A closed Riemannian manifold carries no nonconstant positive superharmonic functions.

Proof. If $h$ is positive superharmonic, then $u:=\ln h$ satisfies $\nabla u+|\Delta u|^{2} \leq 0$. By Stokes' theorem,

$$
\int \Delta u=\int_{\partial} \frac{\partial u}{\partial \vec{n}}=0
$$

since $\partial=\varnothing$. However $\Delta u \leq-|\nabla u|^{2}$, so $\int|\nabla u|^{2} \leq 0$, so $\nabla u \equiv 0$, so $u$ is constant, so $h=e^{u}$ is also constant.

The next step is

Proposition 0.2. A complete finite volume Riemannian manifold carries no nonconstant positive superharmonic functions.

The proof of this proposition is somewhat more complicated because Stokes' theorem only applies to compactly supported forms. Thus it is necessary to introduce a gently sloping cutoff function with compact support. In the case of a single leaf, these functions are easily obtained as radial functions (depending only on the distance to some chosen point). Modulo finding such cutoff functions, the proof of Proposition 0.2 is essentially the same as the proof of the main theorem (Theorem 5.1). The new complication in proving Theorem 5.1 is that ergodicity precludes choosing a single point in each leaf of a foliation, so finding the appropriate cutoff function becomes more difficult. Thus we devote one section ( $(4)$ to proving a result (Lemma 4.7) about cutoff functions. With Lemma 4.7 , the proof of the main theorem is routine.

The foliations we consider here will be the measurable foliations of Zimmer $[Z 1, Z 2]$. These include any $C^{\infty}$-foliation of a manifold, although we specialize here to the case of foliations with a holonomy invariant system of transverse measures and with finite total volume. On manifolds, these kinds of foliations have been extensively studied.

The lack of positive superharmonic functions and the lack of convex functions are basic tools in the study of finite volume manifolds of positive Ricci curvature. It is our hope that these tools can be set up and used in a foliation context as well.

I would like to thank R. Schoen for showing me a proof of Proposition 0.2. It is the basis of this paper. Conversations with A. Freire have been very helpful as well. I would also like to thank G. Stuck for pointing out a number of mistakes in the original version of this paper.

\section{BASIC DEFINITIONS}

All Borel spaces we consider will be standard, i.e., Borel isomorphic to $[0,1]$. A measure space will always be a standard Borel space with a $\sigma$-finite Borel measure.

For the remainder of the paper, we fix a positive integer $n$ (to denote the dimension of the leaves of the foliation we study). Let $D$ denote the unit ball in $\mathbf{R}^{n}$.

If $R$ is an equivalence relation on a set $S$, then, for all $s \in S$, we let $[s]_{R}$ denote the equivalence class of $s$. If $A \subseteq S$, then the $R$-saturation of $A$ is 
denoted $[A]_{R}:=\bigcup_{a \in A}[a]_{R}$. We say that $R$ is countable if every equivalence class of $R$ is countable.

Definition 1.1. If $R$ is a countable equivalence relation on a Borel space $B$, then we say that $R$ is Borel if its graph $R \subseteq B \times B$ is a Borel subset. A Borel automorphism $f: B \rightarrow B$ is called an automorphism of $R$ if $(b, f(b)) \in R$, for all $b \in B$.

Definition 1.2. A countable Borel equivalence relation $R$ on a finite measure space $T$ is said to be measure preserving if every automorphism of $R$ is measure preserving.

Definition 1.3. A flow bow consists of

(i) a countable measure preserving Borel equivalence relation $R$ on a finite measure space $T$; and

(ii) a measurable map $t \rightarrow \omega_{t}$ from $T$ to the space of all Riemannian metrics on $D$. Let $\mu_{t}$ be the volume density associated to $\omega_{t}$, and define a measure $\mu$ on $T \times D$ by

$$
\mu(A):=\int_{T} \mu_{t}\{d \in D \mid(t, d) \in A\} d t,
$$

for all measurable $A \subseteq T \times D$. The resulting measure space is denoted $T \times{ }_{\omega} D$.

In the next definition, no measurability assumption is made on the map $\mathscr{R}$. In fact, Definition 1.5 makes precise what it means for the map $\mathscr{R}$ to be "measurable".

Definition 1.4. Let $\mathscr{F}$ be a Borel equivalence relation on a measure space $M$. Let $\mathscr{R}: L \mapsto \mathscr{R}(L)$ be an association of an $n$-dimensional $C^{\infty}$-Riemannian manifold structure to each equivalence class $L \subseteq M$. A foliation chart for $(M, \mathscr{F}, \mathscr{R})$ consists of

(i) a flow box $\left(T, R, t \mapsto \omega_{t}\right)$; and

(ii) a measure preserving Borel injection $u: T \times{ }_{\omega} D \rightarrow M$ such that (a) for all $d, d^{\prime} \in D$, for all $t, t^{\prime} \in T$,

$$
\left(t, t^{\prime}\right) \in R \Leftrightarrow\left(u(t, d), u\left(t^{\prime}, d^{\prime}\right)\right) \in \mathscr{F},
$$

such that (b), for every equivalence class $L \subseteq M, u(T \times\{0\}) \cap L$ has no accumulation points in the topological space $L$ and such that (c) for all $t \in T$, with $L_{t}:=[u(t, 0)]_{\mathscr{F}}$, the map

$$
d \mapsto u(t, d):\left(D, \omega_{t}\right) \rightarrow\left(L_{t}, \mathscr{R}\left(L_{t}\right)\right)
$$

is an isometry.

Definition 1.5. Let $\mathscr{F}$ be a Borel equivalence relation on a finite measure space $M$. Let $\mathscr{R}$ assign an $n$-dimensional $C^{\infty}$-Riemannian manifold structure to each equivalence class of $\mathscr{F}$. Then $(M, \mathscr{F}, \mathscr{R})$ is a finite volume oriented measurable Riemannian foliation if there exists a countable collection $\mathscr{A}:=$ $\left\{\left(T_{i}, R_{i}, t \mapsto \omega_{t}^{i}, u_{i}\right)\right\}_{i=1,2, \ldots}$ of foliation charts such that

(i) $\bigcup_{i} u_{i}\left(T_{i} \times R_{i}\right)$ contains a.e. equivalence class; and

(ii) for every $i$, for every $t \in T_{i}, u_{i}(t, \cdot)$ is an orientation preserving $C^{\infty}$-isometry of $\left(D, \omega_{t}^{i}\right)$ onto an open subset of the Riemannian manifold $\left[u_{i}(t, 0)\right]_{\mathscr{F}}$.

Such a collection $\mathscr{A}$ is called an atlas. Equivalence classes are called leaves. 
Now, if $f$ is a function on $M$, then it makes sense to speak of $f$ being $C^{\infty}$ along leaves. For such functions, we have a well-defined leafwise gradient $\nabla f$ and a leafwise Laplacian $\Delta f$.

\section{PARTITIONS OF UNITY} tion.

Let $(M, \mathscr{F}, \mathscr{R})$ be a finite volume oriented measurable Riemannian folia-

Definition 2.1. If $\mathscr{A}=\left\{\left(T_{i}, R_{i}, t \mapsto \omega_{t}^{i}, u_{i}\right)\right\}_{i}$ is an atlas, then a sequence $f_{i}: M \rightarrow[0,1]$ is called a a locally finite partition of unity subordinate to $\mathscr{A}$ if

(i) $\sum_{i} f_{i}=1$ a.e. on $M$;

(ii) each $f_{i}$ is measurable on $M$ and $C^{\infty}$ along leaves;

(iii) for all $i, f_{i}=0$ on $M \backslash u_{i}\left(T_{i} \times D_{i}\right)$;

(iv) for all $i$, for all $t \in T_{i}, d \mapsto f_{i}(t, d): D \rightarrow \mathbf{R}$ has compact support.

(v) if $L$ is a leaf and $K \subseteq L$ is compact, then $f_{i} \mid K \equiv 0$, for all but finitely many $i$.

Lemma 2.2. There exists an atlas $\mathscr{A}$ for $(M, \mathscr{F}, \mathscr{R})$ which has a locally finite partition of unity subordinate to it.

Proof. Let $\mathscr{B}=\left\{\left(U_{j}, S_{j}, t \mapsto \alpha_{t}^{j}, v_{j}\right)\right\}_{j=1,2, \ldots}$ be an atlas for $(M, \mathscr{F}, \mathscr{R})$.

Let $g_{k}: D \rightarrow[0,1], k=1,2, \ldots$, be a locally finite partition of unity for the open ball $D \subseteq \mathbf{R}^{n}$ such that every $g_{k}$ has compact support. (Recall that "local finiteness" means that, for any compact $K \subseteq D, g_{k} \mid D \equiv 0$, for all but finitely many $k$.)

Let $r_{1}, r_{2}, \ldots$ be a strictly increasing sequence of positive real numbers tending toward 1. For $l=1,2, \ldots$, let $D_{l} \subseteq D$ be the open ball about 0 of radius $r_{l}$. For each $l$, let $h_{l}: D \rightarrow[0,1]$ be a $C^{\infty}$ function satisfying $h_{l} \equiv 0$ on $D_{l}$ and $h_{l} \equiv 1$ on $D \backslash D_{l+1}$.

Let $\pi_{2}^{j}: T_{j} \times D \rightarrow D$ denote projection onto the second coordinate. For all positive integers $j, k, l$, define $g_{k}^{j}, h_{l}^{j}: M \rightarrow[0,1]$ by

$$
\begin{aligned}
& g_{k}^{j}(m):= \begin{cases}\left(g_{k} \circ \pi_{2}^{j} \circ v_{j}^{-1}\right)(m), & \text { if } m \in v_{j}\left(U_{j} \times D_{j}\right), \\
0, & \text { otherwise, }\end{cases} \\
& \dot{n}_{l}^{j}(m):= \begin{cases}\left(h_{l} \circ \pi_{2}^{j} \circ v_{j}^{-1}\right)(m), & \text { if } m \in v_{j}\left(U_{j} \times D_{j}\right), \\
1, & \text { otherwise. }\end{cases}
\end{aligned}
$$

Then, for all $m \in M$, there exists $j, k$ such that $g_{k}^{j}(m) \neq 0$. However, for some values of $m$, there might exist infinitely many such pairs $(j, k)$.

For all $j, k$, let $\bar{g}_{k}^{j}:=g_{k}^{j} h_{1}^{j-1} h_{2}^{j-2} \ldots h_{j-1}^{1}$. Then for all $m \in M$ there exists $j, k$ such that $\bar{g}_{k}^{j}(m) \neq 0$. Further, around each $m$, there is a neighborhood $N$ in the topological space $[m]_{\mathscr{F}}$ such that $\bar{g}_{k}^{j} \mid N \equiv 0$ for all but finitely many pairs $(j, k)$. Thus, for every compact subset $K$ in any leaf, $\bar{g}_{k}^{j} \mid K \equiv 0$, for all but finitely many $(j, k)$.

We now renumber. Let $i \mapsto(\varphi(i), \psi(i)): \mathbf{Z} \rightarrow \mathbf{Z}^{2}$ be a bijection. Define

$$
\begin{aligned}
& \bar{f}_{i}:=g_{\psi(i)}^{\varphi(i)}, \quad T_{i}:=U_{\varphi(i)}, \quad R_{i}:=S_{\varphi(i)}, \\
& t \mapsto \omega_{t}^{i}:=t \mapsto \alpha_{t}^{\varphi(i)}, \quad u_{i}:=v_{\varphi(i)} .
\end{aligned}
$$

Let $\mathscr{A}:=\left\{\left(T_{i}, R_{i}, t \mapsto \omega_{t}^{i}, u_{i}\right)\right\}_{i}$ and normalize: let $f_{i}:=\bar{f}_{i} / \sum_{i} \bar{f}_{i}$. 


\section{FORMS}

Definition 3.1. If $\zeta$ is a $k$-form on a Riemannian manifold $L$, then, for all $l \in L$, we define

$$
\|\zeta\|_{l}:=\max \left|\left\langle\zeta, e_{1} \wedge \cdots \wedge e_{k}\right\rangle_{l}\right|
$$

where $e_{1}, \ldots, e_{k}$ ranges over orthonormal collections of $k$ vectors in the tangent space $T_{l} L$.

Let $*$ denote the Hodge-* operator on an oriented $n$-dimensional Riemannian manifold $L$. Then, for any $n$-form $\lambda$ on $L$, for any $l \in L$,

$$
\|\lambda\|_{l}=|(* \lambda)(m)|=\left|\left\langle\lambda, e_{1} \wedge \cdots \wedge e_{n}\right\rangle_{l}\right|
$$

for any orthonormal frame $e_{1}, \ldots, e_{n}$ in $T_{l} L$.

Lemma 3.2. If $\eta$ is an (n-1)-form on an n-dimensional Riemannian manifold $L$ and if $\varphi: L \rightarrow \mathbf{R}$ is $C^{\infty}$, then

$$
\|d \varphi \wedge \eta\|_{l} \leq|(\nabla \varphi)(l)| \cdot\|\eta\|_{l},
$$

for all $l \in L$.

Proof. Let $e_{1}, \ldots, e_{n}$ be an orthonormal frame in $T_{l} L$ such that $\left\langle d \varphi, e_{i}\right\rangle_{l}=$ 0 , for all $i=2, \ldots, n$. Then

$$
\begin{aligned}
\|d \varphi \wedge \eta\|_{l} & =\left|\left\langle d \varphi \wedge \eta, e_{1} \wedge \cdots \wedge e_{n}\right\rangle_{l}\right| \\
& =\left|\left\langle d \varphi, e_{1}\right\rangle_{l}\right| \cdot\left|\left\langle\eta, e_{2} \wedge \cdots \wedge e_{n}\right\rangle_{l}\right| \\
& \leq|(\nabla \varphi)(l)| \cdot\|\eta\|_{l} . \square
\end{aligned}
$$

It makes sense to speak of a measurable $k$-form on $(M, \mathscr{F}, \mathscr{R})$, i.e., a system of $k$-forms (one on each leaf) which becomes measurable when pulled back into any flow box. Since all the leaves are oriented, we have a well-defined leafwise Hodge-* operator,

$$
*:\{\text { measurable } k \text {-forms }\} \rightarrow \text { measurable }(n-k) \text {-forms }\} \text {. }
$$

We also have a well-defined leafwise exterior derivative $d$.

Let $(M, \mathscr{F}, \mathscr{R})$ be a finite volume oriented measurable Riemannian foliation. If $\zeta$ is a measurable $k$-form on $(M, \mathscr{F}, \mathscr{R})$ and if $m \in M$, then restricting $\zeta$ to the Riemannian manifold $[m]_{\mathscr{F}}$ gives meaning to $\|\zeta\|_{m}:=$ $\left\|\zeta \mid[m]_{\mathscr{F}}\right\|_{m}$.

Definition 3.3. If $\zeta$ is a measurable $k$-form on $(M, \mathscr{F}, \mathscr{R})$, then we say that $\zeta$ is bounded if there exists a constant $K \in \mathbf{R}$ such that $\|\zeta\|_{m} \leq K$, for all $m \in M$.

If $\lambda$ is a measurable $n$-form on $(M, \mathscr{F}, \mathscr{R})$, then we say that $\lambda$ is integrable if the function $m \mapsto\|\lambda\|_{m}: M \rightarrow \mathbf{R}$ is in $L^{1}(M)$. In this case, we define

$$
\int_{M} \lambda:=\int_{M}(* \lambda) d \mu,
$$

where $\mu$ denotes the measure on $M$.

Since $M$ is a finite measure space, any bounded $n$-form is integrable.

Now let $\left\{f_{i}\right\}_{i=1,2, \ldots}$ be a locally finite partition of unity subordinate to some atlas $\mathscr{A}$ for $(M, \mathscr{F}, \mathscr{R})$, see Lemma 2.2 . 
Lemma 3.4. If $\eta$ is a measurable $(n-1)$-form on $(M, \mathscr{F}, \mathscr{R})$, if $i$ is a positive integer and if $d\left(f_{i} \eta\right)$ is integrable, then

$$
\int_{M} d\left(f_{i} \eta\right)=0 .
$$

Proof. We may pull the form $f_{i} \eta$ back to the $i$ th flow box, integrate along leaves, then integrate the resulting function along the transversal $T_{i}$.

Since $f_{i}$ is compactly supported along the leaves (when pulled back into the $i$ th flow box, see Definition 2.1, condition (iv)), the leafwise integrals are of compactly supported exact $n$-forms. They are therefore all equal to 0 , and the lemma follows.

\section{CutofF FUNCTIONS}

Let $(M, \mathscr{F}, \mathscr{R})$ be a finite volume oriented measurable Riemannian foliation such that a.e. leaf is complete. Let $\mu$ denote the (finite) measure on $M$. For $A \subseteq M$ of positive measure, we define

$$
\mu_{A}(S):=\frac{\mu(S \cap A)}{\mu(A)},
$$

for all measurable $S \subseteq M$.

Definition 4.1. If $\varepsilon>0$ and $A \subseteq M$ has positive measure, then an $(\varepsilon, A)$-cutoff function is a measurable function $\varphi: M \rightarrow[0,1]$ which is $C^{\infty}$ along leaves and which satisfies

(i) $\mu_{A}\{\varphi=1\}>1-\varepsilon$; and

(ii) $|\nabla \varphi|<\varepsilon$ on $M$.

The product rule implies that the product of an $(\varepsilon, A)$-cutoff function and an $\left(\varepsilon^{\prime}, A\right)$-cutoff function is an $\left(\varepsilon+\varepsilon^{\prime}, A\right)$-cutoff function.

If $S \subseteq M$ and $m \in M$, then we define

$$
d(m, S):=\inf \left\{d_{m}(m, s) \mid s \in S \cap[m]_{\mathscr{F}}\right\},
$$

where $d_{m}$ denotes distance in the Riemannian manifold $[\mathrm{m}]_{\mathscr{F}}$. If $S \cap[\mathrm{m}]_{\mathscr{F}}=$ $\varnothing$, then we set $d(m, S)=+\infty$.

Fix, for the remainder of this section, a $C^{\infty}$ function $s: \mathbf{R} \rightarrow \mathbf{R}$ such that

(i) $s(t)=0$, for all $t \leq 1$;

(ii) $s(t)=1$, for all $t \geq 3$; and

(iii) $0 \leq s^{\prime}(t)<0.98$, for all $t \in \mathbf{R}$.

Extend $s$ to $\mathbf{R} \cup\{+\infty\}$ by setting $s(+\infty)=1$.

Lemma 4.2. If $S \subseteq M$ is measurable and $\varepsilon>0$, then there exists a measurable, leafwise $C^{\infty}$ function $\varphi: M \rightarrow \mathbf{R}$ such that:

(a) $\varphi \mid S \equiv 0$;

(b) $|\nabla \varphi|<\varepsilon$ on $M$; and

(c) $\varphi(m)=1$ for all $m \in M$ satisfying $d(m, S)>4 / \varepsilon$.

Proof. Let $\psi: M \rightarrow \mathbf{R}$ be defined by $\psi(m):=s(\varepsilon d(m, S))$. Then $\psi$ satisfies conclusions (a) and (c). In fact, $\psi$ satisfies

$\left(\mathrm{a}^{\prime}\right) \quad \psi(m)=0$, for all $m \in M$ satisfying $d(m, S)<1 / \varepsilon$; and

(c') $\psi(m)=1$, for all $m \in M$ satisfying $d(m, S)>3 / \varepsilon$. 
Unfortunately $m \mapsto d(m, S)$ is not differentiable, so we cannot just set $\varphi=\psi$. However $m \mapsto d(m, S j)$ is leafwise Lipschitz of norm 1 , and so $\psi$ is leafwise Lipschitz of norm $<(.98) \varepsilon$. We now smooth $\psi$ in vach leaf of each flow box and sum over a partition of unity to get $\varphi$ :

Let $b_{1}, b_{2}, \cdots \in C_{C}^{\infty}\left(\mathbf{R}^{n}\right)$ be a sequence of "bump" functions approximating the Dirac mass at 0 . Specifically, we assume

(A) for all $k, b_{k} \geq 0$ on $\mathbf{R}^{n}$ and $\int b_{k}=1$; and

(B) $r_{k}:=\max \left\{d_{\mathbf{R}^{n}}(0, p) \mid p \in \operatorname{supp} b_{k}\right\}$ satisfies $r_{k}<1 / \varepsilon$, for all $k$, and also satisfies $r_{k} \rightarrow 0$ as $k \rightarrow \infty$.

Let $\left\{f_{i}\right\}_{i}$ and $\mathscr{A}:=\left\{\left(T_{i}, R_{i}, t \mapsto \omega_{t}^{i}, u_{i}\right)\right\}_{i}$ be as in Lemma 2.2. Let $\psi_{i}^{t}(d):=\psi\left(u_{i}(t, d)\right)$, for all $i, t \in T_{i}, d \in D$. For all $m \in M$, define

$$
J(m):=\left\{i \mid f_{i}(m) \neq 0\right\}, \quad n(m):=|J(m)|,
$$

where $|\cdot|$ denotes cardinality.

Fix $i$ and $t \in T_{i}$. Let $D^{\prime}:=\left\{d \in D \mid f_{i}\left(u_{i}(t, d)\right)=0\right\}$. Then $D^{\prime}$ is compact. Let $\omega:=\omega_{t}^{i}$. Let $d_{\omega}$ denote the distance function in $(D, \omega)$ and let $\nabla_{\omega}$ denote the gradient on $(D, \omega)$. Let

$$
N:=\max \left\{n\left(u_{i}\left(d^{\prime}, t\right)\right) \mid d^{\prime} \in D^{\prime}\right\}, \quad G:=\max \left\{\mid \nabla_{\omega} f_{i}\left(u_{i}\left(d^{\prime}, t\right)\right) \| d^{\prime} \in D^{\prime}\right\} .
$$

Since $\left\{f_{i}\right\}_{i}$ is locally finite, $N<\infty$.

Fix all $x, y \in D^{\prime}$ and fix $v \in \mathbf{R}^{n}$ such that $\|v\|=1$. Then, for all $\eta>0$, there exists $\delta>0$ such that

$$
0<t<\delta \Rightarrow x+t v, y+t v \in D, \quad \frac{d_{\omega}(x+t v, y+t v)}{d_{\omega}(x, y)}<1+\eta .
$$

By compactness of $D^{\prime}$ and of the unit sphere in $\mathbf{R}^{n}$, we may, given $\eta$, choose $\delta$ independent of $x, y$ and $v$. Taking $\eta=1 / 98$, we conclude that there exists $\delta_{0}>0$ such that

$$
x, y \in D^{\prime},\|v\|<\delta_{0} \Rightarrow x+v, y+v \in D, \quad \frac{d_{\omega}(x+v, y+v)}{d_{\omega}(x, y)}<\frac{99}{98} .
$$

Let $k$ be the smallest positive integer satisfying

(C) $D^{\prime}+\operatorname{supp} b_{k} \subseteq D$;

(D) if $x, y \in D^{\prime}$ and $v \in \operatorname{supp} b_{k}$, then

$$
\frac{d_{\omega}(x+v, y+v)}{d_{\omega}(x, y)}<\frac{99}{98}
$$

and

(E) $\varphi_{t}^{i}:=\psi_{t}^{i} * b_{k}: D^{\prime} \rightarrow \mathbf{R}$ satisfies $\left|\varphi_{t}^{i}-\psi_{t}^{i}\right|<\varepsilon / 100 G N$ on $D^{\prime}$.

Note: (C) and (D) above are obtained by choosing $k$ so large that $r_{k}<\delta_{0}$.

Now fix $i$ and let $t \in T_{i}$ be variable. Define $\varphi_{i}: M \rightarrow \mathbf{R}$ by $\varphi_{i}\left(u_{i}(t, d)\right):=$ $\varphi_{t}^{i}(d)$ and $\varphi_{i} \equiv 0$ on $M \backslash u_{i}(T \times D)$. Since $\psi$ is leafwise Lipschitz of norm $<(.98) \varepsilon$, it follows from (D) that $\varphi_{i}$ is leafwise Lipschitz of norm $<(.99) \varepsilon$. Further, $\varphi_{i}$ is leafwise $C^{\infty}$, by standard properties of convolution, so $\left|\nabla \varphi_{i}\right|<$ (.99) $\varepsilon$ on $M$. Finally, from (E), we see that, for all $m \in \operatorname{supp} f_{i}$,

$$
\left|\varphi_{i}(m)-\psi(m)\right|<\frac{\varepsilon}{100\left|\nabla f_{i}(m)\right| n(m)} .
$$

Let $\varphi:=\sum f_{i} \varphi_{i}$. Now $\sum f_{i} \equiv 1$, so $\sum \nabla f_{i} \equiv 0$, so $\sum\left(\nabla f_{i}\right) \psi \equiv 0$ on $M$. From this and the product rule,

$$
\nabla \varphi \equiv \sum\left(\nabla f_{i}\right)\left(\varphi_{i}-\psi\right)+\sum f_{i} \nabla \varphi_{i}
$$


on $M$. Since $f_{i} \geq 0$, we have $f_{i}(m)=0 \Rightarrow\left(\nabla f_{i}\right)(m)=0$. Taking absolute values and using the estimate (1), we have, for all $m \in M$,

$$
\begin{aligned}
|\nabla \varphi(m)| & \leq \sum_{i \in J(m)}\left|\nabla f_{i}(m)\right| \frac{\varepsilon}{\left|100 \nabla f_{i}(m)\right| n(m)}+\sum_{i}\left[f_{i}(m)\right][(.99) \varepsilon] \\
& \leq(.01) \varepsilon+(.99) \varepsilon=\varepsilon .
\end{aligned}
$$

Thus conclusion (b) is satisfied.

By (B), every $r_{k}<1 / \varepsilon$, so conclusion (a) follows from $\left(a^{\prime}\right)$. Similarly, conclusion (c) follows from $\left(\mathrm{c}^{\prime}\right)$.

For the remainder of this section fix $\varepsilon>0$ and fix some $A \subseteq M$ of positive measure.

Lemma 4.3. Let $F: M \rightarrow \mathbf{R}$ be measurable and bounded on any compact subset of any leaf. Then there exists a constant $K \in \mathbf{R}$ and an $(\varepsilon, A)$-cutoff function $\varphi$ such that $\varphi F \equiv 0$ on $\{|F|>K\}$. In particular, $\varphi F$ is bounded.

Proof. Let $G(m):=\sup \left\{F\left(m^{\prime}\right) \mid m^{\prime} \in \bar{B}(m, 4 / \varepsilon)\right\}$, where $\bar{B}(m, r)$ denotes the closed ball of radius $r$ about $m$ in the Riemannian manifold $[m]_{\mathscr{F}}$. (Since a.e. leaf is complete, closed balls are a.e. compact, so $G$ is finite a.e.)

Since $G$ is measurable, choose $K$ so large that

$$
\mu_{A}\{G \leq K\} \geq 1-\varepsilon .
$$

Let $S:=\{|F|>K\}$ and apply Lemma 4.2.

Corollary 4.4. If $\zeta$ is a measurable $k$-form on $(M, \mathscr{F}, \mathscr{R})$, then there exists an $(\varepsilon, A)$-cutoff function $\varphi$ such that $\varphi \zeta$ is bounded.

Proof. Let $F(m):=\|\eta\|_{m}$ in Lemma 4.3.

Corollary 4.5. Let $\zeta_{1}, \ldots, \zeta_{N}$ be measurable forms on $(M, \mathscr{F}, \mathscr{R})$. Then there exists an $(\varepsilon, A)$-cutoff function $\varphi$ such that $\varphi \zeta_{1}, \ldots, \varphi \zeta_{N}$ are all bounded. Proof. By Corollary 4.4, we may choose for each $i$, an $(\varepsilon / N, A)$-cutoff function $\varphi_{i}$ such that $\varphi_{i} \zeta_{i}$ is bounded. By the product rule, $\varphi:=\varphi_{1} \ldots \varphi_{N}$ is an $(\varepsilon, A)$ cutoff function. The product of a bounded function and a bounded form is a bounded form, so this $\varphi$ has the required properties.

Let $\left\{f_{i}\right\}_{i=1,2}, \ldots$ be a locally finite partition of unity subordinate to an atlas $\mathscr{A}$ for $(M, \mathscr{F}, \mathscr{R})$, see Lemma 2.2 .

Corollary 4.6. Let $\zeta$ be a measurable $k$-form on $(M, \mathscr{F}, \mathscr{R})$. Then there exists an $(\varepsilon, A)$-cutoff function $\varphi$ such that $\varphi f_{i} \zeta \equiv 0$ on $M$, for all but finitely many $i$.

Proof. Let $F(m):=\max \left\{i \mid f_{i}(m) \neq 0\right\}$. Since the partition of unity $\left\{f_{i}\right\}_{i}$ is locally finite (see Definition $2.1(\mathrm{v})$ ), this function is bounded on compact subsets of leaves, and so we may apply Lemma 4.3.

We now come to the main technical result that we will need:

Lemma 4.7. Let $A \subseteq M$ have positive measure, let $\varepsilon>0$ and let $\eta$ be a measurable $(n-1)$-form on $(M, \mathscr{F}, \mathscr{R})$. Then there exists an $(\varepsilon, A)$-cutoff function $\varphi$ such that

(i) $\varphi d \varphi \wedge \eta$ is bounded;

(ii) $\varphi^{2} d \eta$ is bounded; 
(iii) $d\left(\varphi^{2} \eta\right)$ is bounded (hence integrable); and

(iv) $\int_{M} d\left(\varphi^{2} \eta\right)=0$.

Proof. By Corollary 4.6 , choose an $(\varepsilon / 2, A)$-cutoff function $\psi$ and a positive integer $N$ such that $\psi f_{i} \eta \equiv 0$ on $M$, for all $i>N$.

By Corollary 4.5 , choose an $(\varepsilon / 2, A)$-cutoff function $\chi$ such that

(A) $\chi \eta$ is bounded;

(B) $\chi d \eta$ is bounded;

(C) $\chi\left(f_{i} \eta\right)$ is bounded, for all $i=1, \ldots, N$; and

(D) $\chi d\left(f_{i} \eta\right)$ is bounded, for all $i=1, \ldots, N$.

Let $\varphi=\chi \psi$; by the product rule, $\varphi$ is an $(\varepsilon, A)$-cutoff function. Since the product of a bounded function and a bounded form is a bounded form, we conclude

$\left(\mathrm{A}^{\prime}\right) \varphi \eta=\psi(\chi \eta)$ is bounded;

(B) $\varphi^{2} d \eta=\left(\chi \psi^{2}\right)(\chi d \eta)$ is bounded;

(C') $\varphi\left(f_{i} \eta\right)=\psi\left(\chi f_{i} \eta\right)$ is bounded, for all $i=1, \ldots, N$; and

( $\left.\mathrm{D}^{\prime}\right) \varphi^{2} d\left(f_{i} \eta\right)=\left(\chi \psi^{2}\right)\left(\chi d\left(f_{i} \eta\right)\right)$ is bounded, for all $i=1, \ldots, N$.

By definition of a cutoff function (Definition 4.1), the function $|\nabla \varphi|$ is bounded. Thus, by Lemma 3.2, if $\zeta$ is any bounded measurable $(n-1)$-form, then $d \varphi \wedge \zeta$ is also bounded. Thus

$\left(\mathrm{A}^{\prime \prime}\right) \varphi d \varphi \wedge \eta=d \varphi \wedge(\varphi \eta)$ is bounded, for all $i=1, \ldots, N$; and

$\left(\mathrm{C}^{\prime \prime}\right) \varphi d \varphi \wedge\left(f_{i} \eta\right)=d \varphi \wedge\left(\varphi f_{i} \eta\right)$ is bounded, for all $i=1, \ldots, N$.

Now conclusion (i) is just $\left(A^{\prime \prime}\right)$ and conclusion (ii) is $\left(B^{\prime}\right)$. Conclusion (iii) is a linear combination of (i) and (ii), since

$$
d\left(\varphi^{2} \eta\right)=2 \varphi d \varphi \wedge \eta+\varphi^{2} d \eta
$$

By exactly the same argument, $\left(\mathrm{C}^{\prime \prime}\right)$ and $\left(\mathrm{D}^{\prime}\right)$ imply that the form

$$
d\left(\varphi^{2} f_{i} \eta\right)=2 \varphi d \varphi \wedge\left(f_{i} \eta\right)+\varphi^{2} d\left(f_{i} \eta\right)
$$

is bounded, for $i=1, \ldots, N$. As bounded implies integrable, Lemma 3.4 yields

$$
\int_{M} d\left(\varphi^{2} f_{i} \eta\right)=0
$$

for all $i=1, \ldots, N$. On the other hand, by the choice of $\psi$, we have for all $i>N$ that $\varphi f_{i} \eta=\chi\left(\psi f_{i} \eta\right) \equiv 0$ on $M$. Thus conclusion (iv) follows from summing (1) over $i=1, \ldots, N$.

\section{HARMONIC FUNCTIONS}

We now state and prove the main theorem of this paper.

Theorem 5.1. Let $(M, \mathscr{F}, \mathscr{R})$ be a finite volume oriented measurable Riemannian foliation such that a.e. leaf is complete. Let $h: M \rightarrow(0, \infty)$ be a measurable function such that, for a.e. leaf $L \subseteq M$, the function $h \mid L$ is superharmonic on the Riemannian manifold $L$. Then $h \mid L$ is constant, for a.e. leaf $L \subseteq M$.

Proof. Let $u:=\ln h$ and let $\eta:=* d u$, where $*$ denotes the leafwise Hodge-* operator. Then $\Delta u+|\nabla u|^{2} \leq 0$ a.e. on $M$.

Assume for a contradiction that there exists some $\alpha>0$ such that

$$
A:=\{|\nabla u|>\alpha\}
$$


has positive measure. Let $\varepsilon>0$ be arbitrary. Choose $\varphi$ as in Lemma 4.7. Then

$$
\int_{M} 2 \varphi d \varphi \wedge \eta+\varphi^{2} d \eta=\int_{M} d\left(\varphi^{2} \eta\right)=0 .
$$

From this and conclusion (i) of Lemma 4.7,

$$
-\int_{M} \varphi^{2} d \eta=\int_{M} 2 \varphi d \varphi \wedge \eta<\infty .
$$

Let $\lambda$ denote the (oriented) volume $n$-form on the leaves of $\mathscr{F}$, i.e., $\lambda:=* 1$. Then,

a.e. on $M$, and

$$
* d \eta=* d * d u=\Delta u \leq-|\nabla u|^{2},
$$

$$
*(d \varphi \wedge \eta)=*(d \varphi \wedge(* d u))=\nabla \varphi \cdot \nabla u .
$$

Thus (1) becomes

$$
\int_{M} \varphi^{2}|\nabla u|^{2} d \mu \leq \int_{M} 2 \varphi \nabla \varphi \cdot \nabla u d \mu<\infty,
$$

where $\mu$ denotes the measure on $M$.

Now $2 a b \leq 2(a / \sqrt{2})(b \sqrt{2})$, so

$$
2 a b \leq\left(\frac{a}{\sqrt{2}}\right)^{2}+(b \sqrt{2})^{2}=\frac{a^{2}}{2}+2 b^{2} .
$$

Letting $a:=\varphi|\nabla u|$ and $b:=|\nabla \varphi|$, we obtain

$$
2 \varphi \nabla \varphi \cdot \nabla u \leq 2(\varphi|\nabla u|)|\nabla \varphi| \leq \frac{\varphi^{2}|\nabla u|^{2}}{2}+2|\nabla \varphi|^{2} .
$$

Plugging this into $(2)$ yields

$$
\int_{M} \varphi^{2}|\nabla u|^{2} d \mu \leq \frac{1}{2} \int_{M} \varphi^{2}|\nabla u|^{2} d \mu+2 \int_{M}|\nabla \varphi|^{2} d \mu .
$$

By (2), $\frac{1}{2} \int_{M} \varphi^{2}|\nabla u|^{2} d \mu<\infty$, so we may subtract this quantity from both sides of (3) and obtain

$$
\frac{1}{2} \int_{M} \varphi^{2}|\nabla u|^{2} d \mu \leq 2 \int_{M}|\nabla \varphi|^{2} d \mu .
$$

Let $B:=\{\varphi=1\} \cap A$. Since $\varphi$ is an $(\varepsilon, A)$-cutoff function (Definition 4.1),

$$
(1-\varepsilon) \mu(A)<\mu(B) \text { and }|\nabla \varphi|^{2}<\varepsilon^{2} .
$$

Since $B \subseteq A=\left\{\alpha^{2}<|\nabla u|^{2}\right\}$,

So, by (4),

$$
\begin{aligned}
& (1-\varepsilon) \alpha^{2} \mu(A)<\alpha^{2} \mu(B)<\int_{B} \alpha^{2} d \mu \\
& \quad<\int_{B}|\nabla u|^{2} d \mu=\int_{B} \varphi^{2}|\nabla u|^{2} d \mu \leq \int_{M} \varphi^{2}|\nabla u|^{2} d \mu .
\end{aligned}
$$

$$
\frac{(1-\varepsilon) \alpha^{2} \mu(A)}{2}<2 \int_{M}|\nabla \varphi|^{2} d \mu<2 \int_{M} \varepsilon d \mu=2 \varepsilon^{2} \mu(M),
$$

for all $\varepsilon>0$. As $\mu(A)>0$ and $\alpha>0$, this is a contradiction, for $\varepsilon$ sufficiently small.

Thus $|\nabla u|=0$ a.e. on $M$, so, by continuity, $u$ is constant on a.e. leaf. So the same must be true of $h=e^{u}$. 


\section{REFERENCES}

[G] L. Garnett, Foliations, the ergodic theorem and Brownian motion, J. Funct. Anal. 51 (1983), 285-311.

[Z1] R. Zimmer, Ergodic theory, semisimple Lie groups and foliations by manifolds for negative curvature, Publ. Math. IHES 55 (1982), 37-62.

[Z2] _ C Curvature of leaves in amenable foliations, Amer. J. Math. 105 (1983), 1011-1022.

Department of Mathematics, Stanford University, Stanford, California 94305

Current address: Department of Mathematics, University of Chicago, Chicago, Illinois 60637

E-mail address: adams@math.uchicago.edu 